\title{
Comment on amplitude modulations in the ultraviolet-photoemission-spectroscopy valence-band spectra of $\mathrm{Au}$ and $\mathrm{Ag}$
}

\author{
J. Barth \\ II. Institut für Experimentalphysik, Universität Hamburg, 2000 Hamburg 50, Germany
}

(Received 4 March 1980)

\begin{abstract}
Modulations of the relative peak heights of the $d$-band maxima previously observed in ultraviolet photoelectron spectra of $\mathrm{Au}$ and $\mathrm{Ag}$ are shown to correspond to the thresholds of excitations from inner levels. Based on this finding an explanation of the modulations as an effect of atomic electron correlation interaction is proposed.
\end{abstract}

In a previous publication in 1976 Lindau et al. reported photoemission measurements on polycrystalline gold films in the energy range 30-300 eV. As one result they showed that an unexpected modulation of the relative amplitudes of the two maxima in the valence band occurs for photon energies between 100 and $200 \mathrm{eV}$. The authors had no satisfactory explanation for this effect. ${ }^{1}$ It was held most likely that differences in the energy dependence of the photoionization cross sections for the two spin-orbit components, $5 d_{5 / 2}$ and $5 d_{3 / 2}$, are responsible for the observed modulation. In a later publication Stöhr et al. took up this discussion on the basis of their angleresolved photoemission measurements of the $\mathrm{Au}$ valence band. ${ }^{2}$ Since they found a similar effect only for the (111) surface but not for the (001) surface, they excluded the explanation of Lindau et al. and stated that band-structure-induced cross-section effects in connection with a preferential orientation of the crystallites in evaporated Au films might have been responsible for the above-mentioned effect. ${ }^{2}$

In this paper the observed amplitude modulation is related to older measurements of Freeouf $e t$ $a l$. in the energy range below $100 \mathrm{eV}^{3}$ As a result, electron correlations in the different subshells of the atom are proposed as an explanation of the modulation. This is supported by a reinterpretation of a similar effect observed for Ag leading to a corresponding result.

Freeouf et al. published in 1973 a set of energy distribution curves (EDC's) from the Au valence band for excitation energies below $100 \mathrm{eV} .^{3}$ The comparison with an $\mathrm{x}$-ray photoemission spectrum led the authors to the conclusion that the "x-ray limit" from which on the spectra resemble the density of initial states is reached at around $30 \mathrm{eV}$ for $\mathrm{Au}$. However small changes in the spectra with photon energy have also been noted. ${ }^{3}$ Their experimental results have been confirmed by Lindau et al. ${ }^{1}$ Nevertheless, no attempt has been made to analyze the relative peak heights of the valence-band maxima from the EDC's presented by Freeouf et al. and to connect them to the above-mentioned results for photon energies above $100 \mathrm{eV}$. This is done in Fig. 1 showing rich structure over the entire energy range. Three pronounced modulations are to be seen and they set in at the thresholds of the $5 p$ and $5 s$ excitations. While the intensity ratio in Fig. 1 increases at the two $5 p$ thresholds, it decreases at the $5 s$ threshold. It should be mentioned that the onset of $4 f$ excitations at $84 \mathrm{eV}$ (Ref. 1) has no significant influence on the relative peak heights, whereas the increasing intensity ratio above $150 \mathrm{eV}$ could be correlated with the delayed onset ${ }^{1}$ of the $4 f \rightarrow \epsilon l$ excitations. The specific influence of each correlation between the different subshells, however, may be determined only by the comparison with a detailed calculation. This explanation is in direct contradiction to the one of Stöhr et al. based on a solid-state effect. Except for the case that one part of the band is enhanced by the same amount by which another part is suppressed, the modulation effect should also show up in the subshell cross section of the valence band. In fact, this cross section measured by Stöhr et al. in the angular integrated mode ${ }^{2}$ is in excellent agreement with theoretical predictions for the Au $5 d$ cross section based on purely atomic calculations ${ }^{6,7}$.

An assignment of the valence-band maxima to the atomic spin-orbit components is implicitly involved in the interpretation of Lindau et al. ${ }^{1}$ as it is in the one presented here. It has been shown that calculated densities of states only agree with the $\mathrm{x}$-ray photoemission spectroscopy (XPS) valence-band spectrum if spin-orbit coupling is included. ${ }^{8}$ The energy separation of the $d$-band maxima is about $3 \mathrm{eV}$ (Ref. 1) while the atomic spin-orbit splitting is $1.5 \mathrm{eV} .{ }^{9}$

In order to clarify the relation between the atomic spin-orbit components and the $d$ band of 


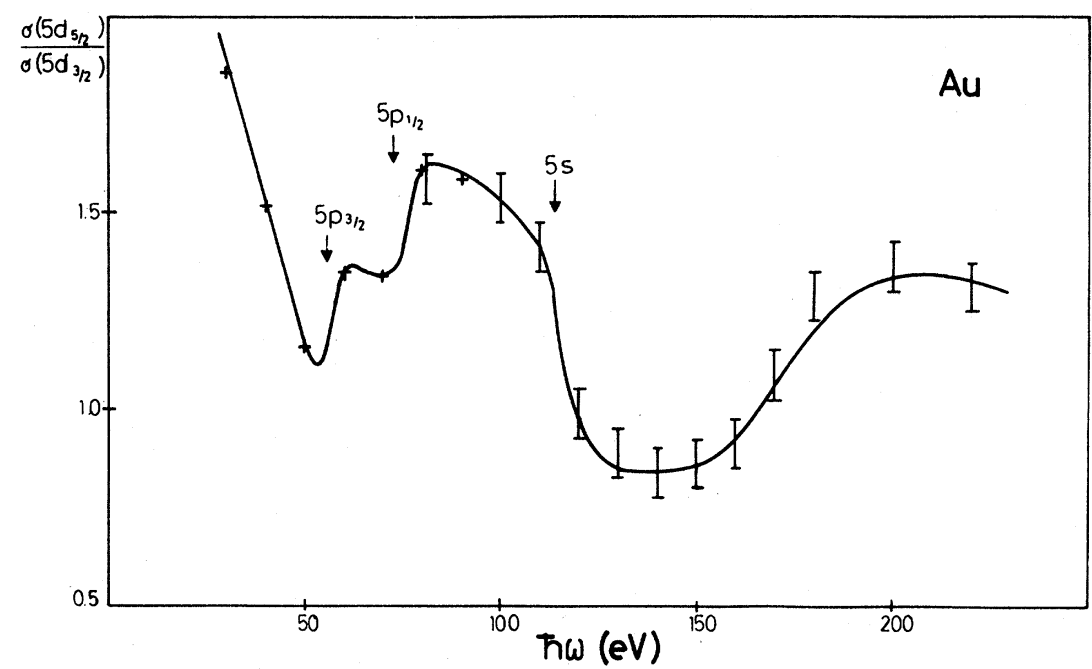

FIG. 1. Ratio between the photoemission peak heights of the low binding energy and the high binding energy maximum in the $\mathrm{Au}$ valence band (after subtraction of linear background) as a function of photon energy. The values are obtained from Ref. $3(+)$ and Ref. 1 (I). The arrows indicate the thresholds of $5 p$ and $5 s$ excitations as given by the binding energies from Ref. $1(5 p)$ and Ref. $4(5 s)$.

solid Au, many attempts have been made to measure the atom-to-solid transition by alloying $\mathrm{Au}$ in different concentrations to other metals..$^{10-14}$ As a consistent result of all of these experiments it was shown that the $d$-band maxima in the solid continually develop out of the spin-orbit components when the interaction between the Au atoms increases. The spin-orbit projected densities of states for the $\mathrm{Au} d$ band confirms the close relationship between the $5 d_{5 / 2}$ and $5 d_{3 / 2}$ levels and the $d$-band maxima in solid $\mathrm{Au}^{15}$ It is therefore not surprising that the relative intensity of the two valence-band maxima is sensitive to inner atomic correlation effects.

For the case of Ag a similar amplitude modulation of the two valence-band maxima as observed for $\mathrm{Au}$ has been reported by Wehner et al. ${ }^{5}$ They explained their result as due to the strong decrease of the $4 d$ subshell cross section between 100 and $130 \mathrm{eV}$ leading to an enhancement of the valence-band peak at higher binding energy by a maximum value of $17 \% .^{5}$ A quantitative analysis of the $4 d$-peak heights ratio is presented in Fig. 2. The ratio increases above $60 \mathrm{eV}$ to a maximum of 1.67 at $80 \mathrm{eV}$ and then decreases to a minimum of 0.94 at $120 \mathrm{eV}$ followed by an increase again. This gives a modulation of \pm $28 \%$ relative to the mean value. Although the mechanism discussed in Ref. 5 must be considered for the valence-band peak-height modulation in $\mathrm{Ag}$, it fails to explain the increase of the intensity ratio below $100 \mathrm{eV}$ and it does not give the full magnitude of the modulation above $100 \mathrm{eV}$. On the contrary, the correlation between the modulations and the thresholds of the $4 s$ and $4 p$ core-level excitations is obvious again (see Fig. 2). In fact, the same arguments as above can be applied for $\mathrm{Ag}$. The atomic spinorbit splitting of the $4 d$ level is $0.6 \mathrm{eV}$ (Ref. 8) and the comparison between the XPS valence-band spectrum and calculated density of states revealed the influence of spin-orbit coupling also in the solid. ${ }^{16}$ Again, photoemission measurements of alloys with different concentrations of $\mathrm{Ag}$ demonstrate a similar relation between the atomic spin-orbit components and the $d$-band maxima of the solid ${ }^{17,18}$ and in this respect agree with the spin-orbit projected densities of states..$^{15}$ Furthermore, a comparison of the $4 d$ photoemission from $\mathrm{Pd}$ to $\mathrm{Xe}$ shows that for the case of $\mathrm{Ag}$ band effects and the spin-orbit coupling appear to be equally important. ${ }^{19}$

In conclusion, atomic correlation interaction has to be taken into account for an explanation of the photoemission intensity modulations in the $d$ bands of $\mathrm{Au}$ and $\mathrm{Ag}$. This was demonstrated by the correspondence between the modulation onsets and the thresholds of inner-level excitations. Recent measurements on Pt (Ref. 20), $\mathrm{Ni}$ (Ref. 21), and $\mathrm{Cr}$ (Ref. 22) show that the $5 d$ $(3 d)$ subshell cross section is coupled to excitations from inner shells into the empty $d$ states. While these results point out the importance of the interaction of an incompletely filled outer $d$ shell with inner-core-level excitations, correlation effects have also been found in gaseous $\mathrm{Xe}^{23}$ In this case oscillator strength of the huge $4 d$ absorption maximum is given to the $5 s$ and 


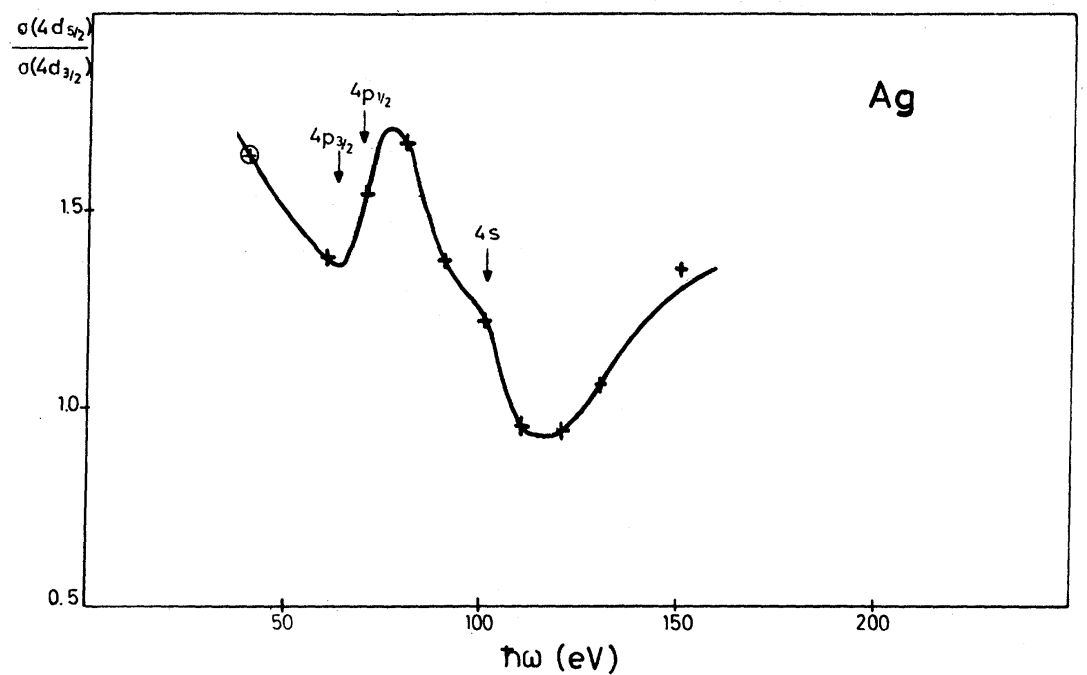

FIG. 2. Ratio between the photoemission peak heights of the two $d$-band maxima in Ag. The values are obtained in the same way as for Au after Ref. $5(+)$ and Ref. $18(\oplus)$. The arrows indicate e thresholds of the $4 p$ and $4 s$ excitations as given by the binding energies from Ref. 4 .

$5 p$ excitation. Very recently the ${ }^{2} P_{3 / 2}:{ }^{2} P_{1 / 2}$ branching ratio of the outermost $p$ shells of atomic $\mathrm{Kr}$ and $\mathrm{Xe}$ have been shown to be dramatically influenced by intershell correlations. ${ }^{24}$ This result stresses the importance of correlation interaction for photoemission spectra in- troduced $b$ inner-level excitations even in closedshell syste is.

I wish to tank C. Kunz for helpful discussions and V.Sai for a critical reading of the manuscript.
${ }^{1}$ I. Lindau, P. Pianetta, K. Yu, and W. E. Spicer, Phys. Rev. B 13, 492 (1976).

${ }^{2}$ J. Stöhr, G. Apai, P. S. Wehner, F. R. McFeely, R. S. Williams, and D. A. Shirley, Phys. Rev. B 14, 5144 (1976).

${ }^{3}$ J. Freeouf, M. Erbudak, and D. E. Eastman, Solid State Commun. 13, 771 (1973).

${ }^{4}$ Handbook of X-Ray and Ultraviolet Photoelectron Spectroscopy, edited by D. Briggs (Heyden, London, 1974).

${ }^{5}$ P. S. Wehner, J. Stöhr, G. Apai, F. R. McFeely, R. S. Williams, and D. A. Shirley, Phys. Rev. B 14, 2411 (1976).

${ }^{6}$ S. T. Manson and J. W. Cooper, Phys. Rev. 165, 126 (1968).

${ }^{7}$ F. Combet Farnoux, Phys. Lett. 38A, 405 (1972).

${ }^{8}$ D. A. Shirley, Phys. Rev. B 5, $4 \overline{709}(1972)$.

${ }^{9}$ C. E. Moore, Atomic Energy Levels, Natl. Bur. Stds. (U.S.) Circ. No. 467 (U.S. GPO, Washington, D.C., 1958).

${ }^{10}$ R. E. Watson, J. Hudis, and M. L. Perlmann, Phys. Rev. B 4, 4139 (1971).

${ }^{11}$ R. M. Friedman, J. Hudis, M. L. Perlmann, and R. E. Watson, Phys. Rev. B 4, 4139 (1971).

${ }^{12}$ J. C. Fuggle, L. M. Watson, D. J. Fabian, and P. R.
Norris, lid State Commun. 13, 507 (1973).

${ }^{13}$ N. J. Sh€ :hik, J. Phys. F 5, 1860 (1975).

${ }^{14}$ K. S. Li r, W. R. Salaneck, and I. A. Aksay, Solid State C imun. 19, 329 (1976).

${ }^{15} \mathrm{~N} . \quad$ ristensen, J. Phys. F 8 , L51 (1978).

${ }^{16}$ S. Hüfner, G. K. Wertheim, N. V. Smith, and M. M. Traum, Solid State Commun. 11, 323 (1972).

${ }^{17}$ S. Hüfner, G. K. Wertheim, and J. H. Wernick, Phys. Rev. B 8,4511 (1973).

${ }^{18}$ N. J. Shevchik and A. Goldman, J. Electron Spectrosc. Relat. Phenom. 631 (1974).

${ }^{19}$ R. A. Pollak, S. Kowalczyk, L. Ley, and D. A. Shirley, Phys. Rev. Lett. 29, 274 (1972).

${ }^{20} \mathrm{G}$. P. Williams, G. J. Lapeyre, J. Anderson, R. E. Dietz, and Y. Yafet, J. Vac. Sci. Technol. 16, 528 (1979).

${ }^{21} \mathrm{~J}$. Barth, G. Kalkoffen, and C. Kunz, Phys. Lett. 74A, 360 (1979).

${ }^{22}$ J. Barth, F. Gerken, K. L. I. Kobayashi, J. H. Weaver, and B. Sonntag, J. Phys. C 13, 1369 (1980).

${ }^{23} \mathrm{~J}$. B. West, P. R. Woodruff, K. Codling, and R. G. Houlgate, J. Phys. B 9, 407 (1976).

${ }^{24}$ W. R. Johnson and K. T. Cheng, Phys. Rev. A 20, 978 (1979). 